\title{
Die Anpassung des Steuerrechts an die politischen Verhältnisse und die gesellschaftlichen Vorstellungen in der Weimarer Republik
}

\subsection{Die verfassungsrechtlichen Grundlagen}

Mit der Weimarer Reichsverfassung (WRV) vom 11. August $1919^{761}$ wurden die letzten absolutistischen Privilegien durch Art. 109 aufgehoben: „Alle Deutschen sind vor dem Gesetze gleich“ (Art. 109 Abs. 1); „Öffentlich-rechtliche Vorrechte oder Nachteile der Geburt oder des Standes sind aufzuheben“" (Art. 109 Abs. 2). Das hier ausformulierte rechtspolitische Gleichheitsgebot wurde in Art. 134 dieser Verfassung für den Bereich der Besteuerung als allgemeine Grundpflicht festgeschrieben: „Alle Staatsbürger ohne Unterschied tragen im Verhältnis ihrer Mittel zu allen öffentlichen Lasten nach Maßgabe der Gesetze bei.“

Es wurden aber damit nicht nur die steuerlichen Privilegien für die Standesherren, sondern zusätzlich alle Arten von unbegründeten steuerlichen Befreiungen und Vergünstigungen für unzulässig erklärt. Entsprechend wurde die verfassungsrechtliche Verankerung des Grundsatzes der Allgemeinheit der Besteuerung überwiegend in Art. 134 WRV gesehen. ${ }^{762}$

761 Verfassung des Deutschen Reichs vom 11.08.1919, http://www.verfassungen.de/de/ de19-33/verf19-i.htm (letzter Zugriff: 01.03.2017).

762 Davidsohn, Lars: Verfassungsrechtliche Würdigung, S. 19. 
Der Rechtswissenschaftler Albert Hensel ${ }^{763}$ vertrat die Auffassung, dass der in Art. 134 WRV formulierte Allgemeinheitsgrundsatz nicht nur die Steuerprivilegien untersagte, sondern auch gebot, dass fehlerhafte und unvollständige Formulierungen nicht zu ungerechtfertigten Steuerfreiheiten führen dürften.

Damit vertrat Hensel den Standpunkt, dass ein Verstoß gegen den Allgemeinheitsgrundsatz auch dann vorlag, wenn einzelne Lebenssachverhalte aufgrund einer unzureichenden Formulierung des Steuertatbestandes keiner Besteuerungspflicht unterworfen wären. ${ }^{764}$ Da Hensel generell jede Steuernorm als Verwirklichung einer vorher erarbeitenden Gerechtigkeitsidee betrachtete, sollten die Steuergesetze entsprechend jederzeit mit dieser übereinstimmen. Aus den Ausführungen Hensels ist zu entnehmen, dass er die rechtstechnische Umsetzung eines als steuerwürdig einzustufenden Sachverhaltes nicht nur unter dem Gesichtspunkt des Allgemeinheitsgrundsatzes, sondern auch im Hinblick auf den Grundsatz einer gleichmäßigen und verhältnismäßigen Besteuerung für unverzichtbar hielt.

Ein so verstandener Grundsatz einer gleichmäßigen Besteuerung verlangt in diesem Sinne aber unbedingt, dass kein Steuerpflichtiger irgend ein verdecktes, auf rechtstechnischen Mängeln des Gesetzes beruhendes Steuerprivileg genießt. ${ }^{765}$

Hensel stellte damit eine bemerkenswerte These auch für die heutige juristische Interpretation des Allgemeinheitsgrundsatzes auf. ${ }^{766}$

Das in Art. 134 WRV enthaltene Gebot, die Steuerlasten auf alle Staatsbürger so zu verteilen, dass diese „im Verhältnis ihrer Mittel“ belastet würden, wurde seinerzeit von der herrschenden Lehre als unverbindlicher rechtspolitischer Programmsatz interpretiert. Auch der Reichsfinanzhof betonte in ständiger Rechtsprechung, dass Art. 134 WRV im Wesentlichen nur eine Richtlinie enthalten habe und dass so lediglich offenbare Verletzungen der in der Vorschrift formulierten materiellen Grundsätze ausgeschlossen gewesen wären. ${ }^{767}$

763 Hensel, Albert: Verfassungsrechtliche Bindungen des Steuergesetzgebers. Besteuerung nach Leistungsfähigkeit - Gleichheit vor dem Gesetz, in: Vierteljahresschrift für Steuerund Finanzrecht 4 (1930), S. 460 f.

764 Ebd., S. 460.

765 Davidsohn, Lars: Verfassungsrechtliche Würdigung, S. 20.

766 Ebd., S.20f.

767 RFHE 27, 322; 29, 111; RFH, Reichssteuerblatt 1931, S. 203; vgl. Davidsohn, Lars: Verfassungsrechtliche Würdigung, S. 40. 
Eine derartige Auslegung des Art. 134 WRV entsprach weder dem Rechtsverständnis von Albert Hensel noch dem von Alfred Huttel. ${ }^{768}$ Nach ihrer Ansicht stand dem Gesetzgeber zwar ein nicht unerhebliches Ermessen bei der Ausgestaltung einzelner Steuergesetze zu, doch durfte er dabei nicht gegen die in Art. 134 WRV verankerten materiellen Grundsätze der Allgemeinheit und Gleichmäßigkeit verstoßen, der Wortlaut dieses Artikels sei insoweit eindeutig. ${ }^{769}$ Art. $134 \mathrm{WRV}$ enthielt damit das verbindliche Gebot, die individuelle Leistungsfähigkeit des Einzelnen angemessen und hinreichend bei der Verteilung der Steuerlasten zu berücksichtigen. Für die am Rande des Existenzminimums lebenden Personen, bei denen regelmäßig keine steuerliche Leistungsfähigkeit vorhanden war, schränkte der in Art. 134 WRV enthaltene Grundsatz der Gleichmäßigkeit der Besteuerung das formale Gebot, alle Staatsbürger zur Beitragspflicht heranzuziehen, automatisch ein. Das in Art. 134 enthaltene Subjekt „alle Staatsbürger“ war durch die Formulierung „im Verhältnis ihrer Mittel" eindeutig konkretisiert. ${ }^{770}$

\subsection{Besteuerungspolitik und Reformen in der Weimarer Republik}

\subsubsection{Sozialpolitisch orientierte Besteuerungspolitik in der Weimarer Republik}

Vor dem Ersten Weltkrieg traten für eine sozialpolitische Besteuerungspolitik offenbar nur die Sozialdemokraten und die von Adolph Wagner beeinflussten Christlich-Sozialen ein. ${ }^{771}$ Nach dem Ersten Weltkrieg hatte sich die Einstellung der bürgerlichen Parteien radikal verändert. Selbst die konservativen und liberalen Parteien befürworteten ausdrücklich eine sozialpolitisch geprägte Steuerpolitik, die auf einen Ausgleich der bestehenden Vermögensverhältnisse gerichtet war. Da viele Staatsbürger einen Großteil ihres Vermögens verloren hatten und nur wenige Personen Gewinne erwirtschaften konnten, waren die durch den Krieg

768 Vgl. Huttel, Alfred: Gleichmäßige Besteuerung nach der Leistungsfähigkeit. Inhalt und Bedeutung des Artikels 134 der Reichsverfassung, Frankfurt am Main 1934.

769 Davidsohn, Lars: Verfassungsrechtliche Würdigung, S. 42.

770 Ebd., S. 42-44.

771 Ebd., S. 289. 
entstandenen Vermögensungleichheiten ein zentrales Thema der politischen Auseinandersetzungen nach $1918 .^{772}$

Die großen bürgerlichen Volksparteien der Weimarer Republik - Deutsche Zentrumspartei, Deutsche Volkspartei und Deutsche Demokratische Partei - die oft an Regierungskoalitionen beteiligt waren, warben regelmäßig für eine gerechtere Einkommens- und Besitzverteilung und hatten in ihren Parteiprogrammen finanz- und steuerpolitische Grundsatzerklärungen abgegeben. Die Deutsche Demokratische Partei verlangte in einem Programm aus dem Jahr 1919 ausdrücklich, „soziales Unrecht in der Verteilung des Besitzes und des Einkommens zu beseitigen ". ${ }^{733}$ Die Deutsche Volkspartei forderte eine steuerliche Besserstellung der Arbeitseinkommen gegenüber den Einkommen aus Vermögen sowie eine ausreichende Berücksichtigung der persönlichen und familiären Verhältnisse. ${ }^{774}$ Noch deutlicher verlangte die Deutsche Zentrumspartei „die schärfste steuerliche Erfassung der hohen Einkommen, der großen Vermögen, des unverdienten Wertzuwachses und der durch den Krieg gemachten Gewinne“.775

Die Sozialdemokratische Partei Deutschlands bekannte sich in ihrem Görlitzer Programm aus dem Jahr 1921 ausdrücklich zu einer Steuersozialisierung und hatte vor, Grund und Boden sowie die Bodenschätze „der kapitalistischen Ausbeutung zu entziehen und in den Dienst der Volksgemeinschaft zu überführen“. Ebenfalls wurden im Parteiprogramm von 1921 eine Steigerung der Steuersätze für große Einkommen und eine besonders hohe Belastung des verschwenderischen und maßlosen Überverbrauchs gefordert. ${ }^{776}$

Matthias Erzberger - der der Deutschen Zentrumspartei angehörte und am 21. Juni 1919 im Kabinett Bauer sein Amt als Reichsfinanzminister antrat - hatte mit seinen Reden maßgebend zu dem Umdenken in der Steuerpolitik beigetragen. In

772 Ebd., S. 289.

773 Deutsche Demokratische Partei, Programm der Deutschen Demokratischen Partei vom Dezember 1919, in: Anschütz, Gerhard u. a. (Hrsg.): Handbuch der Politik, Bd. 6, 3. Aufl., Berlin 1926, S. 343-347.

774 Deutsche Volkspartei: Grundsätze der Deutschen Volkspartei. Beschlossen auf dem Parteitag in Leipzig am 19.10.1918, in: Anschütz, Gerhard u. a. (Hrsg.): Handbuch der Politik, Bd. 6, 3. Aufl., Berlin 1926, S. 332-340.

775 Deutsche Zentrumspartei: Aufruf und Leitsätze des Reichsausschußes der Deutschen Zentrumspartei vom 30.12.1918, in: Anschütz, Gerhard u.a. (Hrsg.): Handbuch der Politik, Bd. 6, 3. Aufl. 1926, S. 329-332.

776 Sozialdemokratische Partei Deutschlands: Görlitzer Programm der Sozialdemokratischen Partei Deutschlands vom 23.09.1921, in: Anschütz, Gerhard u.a. (Hrsg.): Handbuch der Politik, Bd. 6, 3. Aufl., Berlin 1926, S.353-357; Bernstein, Eduard: Das Görlitzer Programm der Sozialdemokratischen Partei Deutschlands, Berlin 1922, S. $44 \mathrm{f}$. 
seinem Grundsatzprogramm im Hinblick auf die Fortbildung des Steuersystems zu einem sozialen und gesellschaftlichen Instrument sagte Erzberger vor der Nationalversammlung in Weimar am 8. Juli 1919:

Gerechtigkeit im gesamten Steuerwesen zu schaffen ist mein oberstes Ziel. Gerechte Steuern stellen eine rasch wirkende vorzügliche Sozialisierung dar; sie treffen alle, sie erfassen jeden nach seiner Leistungsfähigkeit unter ausreichender Berücksichtigung des Familienstandes; sie haben aber vor der restlosen Kommunisierung eines voraus: die private Initiative bleibt bestehen, der Selbsterhaltungstrieb und begründete Eigennutz sucht nach höchster Einnahme, die Sorge für die Familie bringt die Auslösung der höchsten Arbeitsleistung. Der erzielte Überschuß wird aber zum erheblichen Teil wieder im Wege der Steuer für die Volksgemeinschaft abgenommen. Gerechte Steuern müssen die Überkapitalisierung eines Volkes verhindern. Sie tun es dann, wenn gewisse Arten von Einnahmen vorbelastet werden. Das Kapitaleinkommen muß eine erhebliche Vorbelastung vor dem Arbeitseinkommen tragen. ${ }^{777}$

Die soziale Komponente zeigte sich im politischen Werdegang von Matthias Erzberger: 1899 war er maßgeblich an der Gründung der christlichen Gewerkschaften in Mainz beteiligt, und in der Reichstagsfraktion führte er den linken Flügel an. ${ }^{778}$ Dies schlug sich auch in der Gesetzgebung nieder, wie aus der Begründung zum Kapitalertragsteuergesetz von 1920 ersichtlich ist:

Das auf Vermögensbesitz beruhende Einkommen, das sogenannte fundierte Einkommen, sollte stärker belastet werden als das unfundierte Einkommen, das den Zufälligkeiten des Fortbestandes unterworfen und von der Dauer der Arbeitskraft und der Arbeitsgelegenheit abhängig sei. ${ }^{779}$

777 Erzberger, Matthias: Reden zur Neuordnung des deutschen Finanzwesens vom Reichsminister der Finanzen Erzberger, Berlin 1919, S. 5.

778 Sahm, Reiner: 5000 Jahre Steuern, S. 270.

779 Aus der Begründung zum Kapitalertragsteuergesetz vom 29.03.1920 (RGBl S. 345), Verhandlungen der verfassungsgebenden Nationalversammlung, Bd. 340, Anl. Nr. 1625, S. 7; zitiert vom Bundesverfassungsgericht, Urteil vom 27.06.1991, BVerGE 84, 233, Az.: 2 BvR 143/89 Nr. 15; https://www.jurion.de/urteile/bverfg/1991-06-27/2-bvr-1493_89 (Letzter Zugriff: 28.02.2017). 


\subsubsection{Die Erzberger-Reform der Finanzverfassung und des Steuersystems}

Matthias Erzberger ${ }^{780}$ setzte die sozial- und steuerpolitischen Forderungen der Weimarer Parteien nach 1919 nur teilweise um. Sowohl die extrem hohen finanziellen Verpflichtungen, die Deutschland gezwungen war, in den Waffenstillstands- und Friedensverhandlungen mit den Siegermächten einzugehen, als auch die staatliche Fürsorge für die große Anzahl an Kranken und Kriegsverletzten ließen sich letztlich nur erfüllen, wenn das Finanzwesen dazu in der Lage war. Ebenso ließ sich der im Verlaufe des Krieges entstandene verheerende Zustand der Finanzen in Deutschland ${ }^{781}$ nur bereinigen, wenn die Finanzverfassung und das Finanzsystem auf eine neue Grundlage gestellt würden. ${ }^{782}$

Finanzminister Erzberger konnte im Gegensatz zu seinen Vorgängern auf eine verfassungsrechtliche Grundlage bauen. Außerdem hatte er - wie jeder Reichsminister - nach Art. 56 WRV innerhalb der vom Reichskanzler vorgegebenen Richtlinien die eigenverantwortliche Leitung seines Ministeriums inne und damit eine Machtkonzentration, die man als unentbehrliche Voraussetzung für die Durchführung der Reform werten kann. ${ }^{783}$

Die von Matthias Erzberger initiierte Reform der Finanzverfassung und des Steuersystems hatte vier Bestandteile: ${ }^{784}$

Der erste Bestandteil ${ }^{785}$ war die Schaffung der reichseinheitlichen Finanz- und Steuerverwaltung. Dazu bedurfte es zunächst der Herausbildung des Reichsfinanzministeriums aus dem Reichsschatzamt und des strukturellen und personellen Aufbaus dieses Ministeriums. Das geschah unmittelbar nach der Berufung des

780 Auf einer Gedenkveranstaltung im Berliner Bundesfinanzministerium ehrte Bundesfinanzminister Schäuble Matthias Erzberger im Jahr 2011 aus Anlass dessen 90. Todestages und hob dessen „völlig einmalige, unglaubliche“ Leistung hervor. Mit seiner Steuerreform sei ihm ein „elementarer Umbruch“ gelungen; o. V.: Reutlinger Generalanzeiger vom 29.08.2011, auf: http://www.gea.de/region+reutlingen/ueber+die+alb/+einsatz+fuer+kleine+leute.2164499.html (letzter Zugriff: 20.02.2017).

781 Die gesamten Reichsschulden wuchsen bis 1913 auf 4,9 Milliarden Mark; dazu kamen 284 Millionen Mark an „schwebenden Schulden“. Insgesamt hatte sich die Reichsschuld zwischen 1877 und 1914 verdreihundertfacht; Ullmann, Hans-Peter: Der deutsche Steuerstaat, S. 63.

782 Sahm, Reiner: 5000 Jahre Steuern, S. 268.

783 Wallmann, Ferdinand: Die Finanzpolitik des deutschen Reiches nach dem Kriege bis zur Währungsstabilisierung, Köln 1931, S.16.

784 Sahm, Reiner: 5000 Jahre Steuern, S. 269 f.

785 Erzberger, Matthias: Reden, S. $111 \mathrm{f}$. 
Ministers. Mit dem Gesetz vom 10. September 1919 wurde die Reichsfinanzverwaltung geschaffen und ihr die Zuständigkeit für die Verbrauchsabgaben und die Zölle übertragen.

Der zweite Bestandteil ${ }^{786}$ war die Ausrichtung der unter dem Reichsministerium angesiedelten Landesämter und Finanzämter. Die gesetzliche Grundlage dafür bot die Reichsabgabenordnung vom 13. Dezember 1919. Sie war von dem Juristen Enno Becker konzipiert worden, der wie kaum ein anderer die Gesetzgebung, Rechtsprechung und Wissenschaft auf dem Gebiet des Steuerrechts beeinflusste. Es ist bemerkenswert, dass diese Reichsabgabenordnung bis zum Jahre 1976 in Kraft blieb. Sie gewährte einerseits dem Steuerbürger Rechtsschutz und räumte dem Staat andererseits umfassende, obrigkeitsstaatliche Machtbefugnisse ein und bestimmte, dass alle Steuern, die zugunsten des Reiches erhoben wurden, von Reichsbehörden $\mathrm{zu}$ verwalten waren. Damit war die Grundlage für eine zentrale Finanzverwaltung mit den darunter angesiedelten Organen bis hin zu den Finanzämtern begründet. Nach dem Erlass der Reichsabgabenordnung wurden dem Reichsfinanzministerium die 26 Landesfinanzämter und die ca. 1.000 Finanzämter unterstellt.

Der bereits 1918 in München geschaffene Reichsfinanzhof war ein weiterer wichtiger Meilenstein zu einem modernen Steuersystem. Damit zog das Reich die Kompetenz der Rechtsprechung sowohl im Steuerrecht als auch im Steuerstrafrecht an sich und ebnete den Weg zu einer Zentralisierung der Reichsaufsicht. Ab 1922 nahmen Finanzgerichte als erste Instanz im Reich ihre Tätigkeit auf.

Der dritte Bestandteil ${ }^{787}$ war das Landessteuergesetz vom 31. März 1920, in dem die Finanzbeziehungen zwischen der Republik, den Ländern und Gemeinden geregelt wurden. Die Republik, die nun die wichtigsten Abgaben einnahm, hatte für eine ausreichende Finanzierung der Länder und Kommunen sowie für einen Finanzausgleich zwischen den Ländern Sorge zu tragen. Länder und Kommunen, die weiterhin berechtigt waren, verschiedene Abgaben zu erheben, sahen sich nun auf einen gewissen Anteil an den Reichssteuern angewiesen. Damit kehrte sich das bisher herrschende Verhältnis zwischen Reich und Ländern um. Das Reich war nicht mehr der Kostgänger der Einzelstaaten, sondern diese waren auf die Zuweisungen aus den Reichseinnahmen angewiesen. ${ }^{788}$

Ein vierter Bestandteil ${ }^{789}$ der Finanz- und Steuerreform bestand in einer Reihe neuer oder veränderter Steuergesetze. Matthias Erzberger ließ sich bei der Abfassung

786 Ebd., S. $113 \mathrm{f}$.

787 Ebd., S. $115 \mathrm{ff}$.

788 Vgl. hierzu die Ausführungen unter Kapitel 6.4.1 sowie Ullmann, Hans-Peter: Der deutsche Steuerstaat, S. 102.

789 Erzberger, Matthias: Reden, S. $103 \mathrm{ff}$. 
dieser Gesetze von dem Gedanken der Umverteilung von Einkommen zugunsten der weniger Bemittelten leiten. Dabei trug er einerseits der aktuellen Situation auf sozialpolitischem Gebiet Rechnung und legte andererseits als Minister seinem finanz- und steuerpolitischen Wirken eine soziale Komponente zugrunde.

Eine der Hauptsäulen der Erzberger-Reform war die Einkommensteuer. Nach der Gründung des Deutschen Reiches am 18. Januar 1871 bestanden auf deutschem Boden 25 und ab 1911 mit Elsaß-Lothringen 26 Bundesstaaten. Bis zur Einführung der Reichseinkommensteuer vom 29. März 1920 gab es aufgrund dieser politischen Situation 27 verschiedene Einkommensteuergesetze ${ }^{790}$ die sich mehr oder weniger an der preußischen und sächsischen Gesetzgebung orientierten. Es gab jedoch keine einheitlichen Regelungen in Bezug auf den Kreis der Steuerpflichtigen, den Einkommensbegriff, das Existenzminimum, den Steuertarif oder die Steuerbefreiungen, -zuschläge und -berechtigungen.$^{791}$

Das Einkommensteuergesetz vom 29. März 1920 (EStG 1920) war sehr stark von dem enormen Finanzbedarf des Reiches beeinflusst, was seinen Niederschlag in der amtlichen Begründung fand:

Bei der Ausgestaltung des Tarifs war davon auszugehen, dass aus der Einkommensteuer an Erträgen für das Reich herauszuholen war, was ohne Gefährdung des Wirtschaftslebens und der Daseinsmöglichkeit des einzelnen herausgeholt werden kann. ${ }^{792}$

Diese Begründung war jedoch kaum vereinbar mit der von Erzberger herausgegebenen Leitlinie seiner Steuerreform, denn „Gerechtigkeit im gesamten Steuerwesen zu schaffen ist mein oberstes Ziel" ${ }^{\text {" }}{ }^{793}$ Allgemeiner Konsens bestand darüber, dass Kriegsgewinne mehr oder minder vollständig abgeschöpft werden sollten, weitgehende Einigkeit bestand auch darin, dass jeder Steuerpflichtige zur Verminderung

790 Sachsen-Coburg-Gotha als Bundesland besaß für Coburg und für Gotha jeweils ein separates Gesetz; vgl. Popitz, Johannes: Einkommensteuer, S. 439.

791 Statistisches Reichsamt: Die deutsche Einkommensbesteuerung vor und nach dem Kriege (Statistik des Deutschen Reiches, N. F. 312), Berlin 1925, ND Osnabrück 1978, S. 5 .

792 o. V.: Begründung zum Reichseinkommensteuergesetzentwurf vom 29.11.1919, Drucksachen der Nationalversammlung Nr. 1624, in: Finanzarchiv 37/2 (1920), S.591- 631, S. 608 .

793 Erzberger, Matthias: Rede vor der Nationalversammlung am 08.07.1919, in: Ders.: Reden, S. 18. 
der Lasten beitragen musste. Es war also nicht grundsätzlich ungerecht, der Bevölkerung eine enorme steuerliche Belastung zuzumuten. ${ }^{794}$

Die Forderung nach einer gerechten Steuer, die jeden Steuerpflichtigen nach seiner Leistungsfähigkeit ${ }^{795}$ unter besonderer Berücksichtigung seiner persönlichen Verhältnisse erfassen sollte, schlug sich insbesondere in den folgenden Regelungen nieder: ${ }^{796} \$ 12$ EStG 1920 enthielt zahlreiche Einzelfälle, die Einkommensbestandteile steuerfrei stellten und zu einer genaueren Abbildung der Leistungsfähigkeit beitragen sollten. Es wurde eine Haushaltsbesteuerung eingeführt, bei der nicht nur die Einkommen der Ehegatten ( $\$ 16$ EStG 1920), sondern auch die zum Haushalt zählenden minderjährigen Kinder erfasst wurden (\$17 EStG 1920). Ein steuerfreies Existenzminimum ( $\$ 20$ EStG 1920) in Höhe von 1.500 Mark wurde im Steuertarif berücksichtigt. Der Steuertarif war als Stufentarif mit progressivem Verlauf gestaltet. In der Begründung zum Reichseinkommensteuergesetzentwurf vom 29. November 1919 wurde hierzu ausgeführt, dass bei der Aufstellung des Steuertarifs und der ihn beeinflussenden Vorschriften neben der Höhe des Einkommens als wesentlichster Maßstab die wirtschaftliche Leistungsfähigkeit zu berücksichtigen ist. ${ }^{797} \mathrm{Nach} \S 21$ EStG 1920 begann der Steuersatz bei 10 Prozent und stieg zunächst vierzehnmal um 1 Prozent für jede 1.000 Mark mehr an Einkommen bis auf 24 Prozent und vergrößerte sich dann „um je 1 v. H., 5 mal für je 2.000, 5 mal für je 3.000, 10 mal für je 5.000, 5 mal für je 10.000, 2 mal für jede 20.000, 2 mal für jede 30.000, 1 mal für jede 40.000 und vier mal für je $50.000 \mathrm{M}$. Mehreinkommen“ bis auf 59 Prozent. Alle darüber liegenden Einkommensanteile waren mit 60 Prozent zu versteuern. ${ }^{798}$

Gemäß $\$ 26$ Abs. 1 EStG 1920 konnten ,besondere wirtschaftliche Verhältnisse[,] die die Leistungsfähigkeit des Steuerpflichtigen wesentlich beeinträchtigen, berücksichtigt werden, sofern das steuerbare Einkommen den Betrag von dreißigtausend Mark nicht übersteigt." Die nach $\$ \$ 19$ bis 25 zu erhebende Abgabe konnte insbesondere durch außergewöhnliche Belastungen, wie durch den Unterhalt und die Erziehung der Kinder, durch Verpflichtung zum Unterhalte mittelloser Angehöriger, sowie durch Krankheit, Körperverletzung, Verschuldung, Unglücksfälle oder durch

794 Hacker, Mark: Gibt es „Gerechtigkeit“ in der Steuerpolitik? Der politisch-philosophische Diskurs über Recht und Gerechtigkeit am Beispiel der Entstehung des modernen Einkommensteuerrechts in der Weimarer Republik, Stuttgart/Berlin 2013, S. 178.

795 Erzberger, Matthias: Rede vor der Nationalversammlung am 08.07.1919: „Gerechte Steuern ... erfassen jeden nach seiner Leistungsfähigkeit“, in: Ders.: Reden, S. 5.

796 Hacker, Mark: Gibt es „Gerechtigkeit“ in der Steuerpolitik?, S. 158 und S. 182-184.

797 o. V.: Begründung zum Reichseinkommensteuergesetzentwurf, S. 243.

798 Metzger, Ulrike/Weingarten, Joe: Einkommensteuer und Einkommensteuerverwaltung in Deutschland. Ein historischer und verwaltungswissenschaftlicher Überblick, Wiesbaden 1989, S. 138. 
außergewöhnliche Aufwendungen im Haushalt infolge einer Erwerbstätigkeit der Ehefrau ( $\$ 26$ Abs. 2 EStG 1920) ermäßigt werden.

Das Einkommensteuergesetz 1920 wurde durch das Körperschaftsteuergesetz vom 29. März $1920^{799}$ ergänzt, das erstmalig eine Trennung von natürlichen und juristischen Personen vornahm und den Kreis der Steuerpflichtigen auf juristische Personen beschränkte. Aufgrund des verheerenden Zustands der Finanzen in Deutschland ${ }^{800}$ konnte das Reich auf eine Besteuerung der Körperschaften nicht verzichten, denn dies hätte Einnahmenausfälle bedeutet. ${ }^{801}$ In der amtlichen Begründung zum Entwurf des Einkommensteuergesetzes 1920 heißt es: „Die nichtphysischen Personen stellen in der Einkommensteuer einen Fremdkörper dar, der in deren Rahmen nur mit Hilfe von Fiktionen hineingepresst werden kann. “802 Sowohl die Körperschaftsteuer als auch die Einkommensteuer bildeten gemeinsam das Rückgrat der direkten Personalbesteuerung des Reichs ${ }^{803}$ Eine Entwicklung, die mit dem Preußischen Einkommensteuergesetz von 1891 begonnen hatte, fand damit ein Ende. ${ }^{804}$

Der Körperschaftsteuertarif betrug unabhängig von der Gewinnverwendung 10 Prozent des steuerbaren Einkommens. ${ }^{805}$ Die Körperschaftsteuer für ausgeschüttete Gewinne erhöhte sich um einen Zuschlag, der um bis zu $10 \%$ dieser Beträge antieg. Da auch der Einkommensteuertarif angehoben wurde, traten die Wirkungen der Doppelbelastung hervor. ${ }^{806}$ Damit waren Gewinnausschüttungen gegenüber Thesaurierungen deutlich benachteiligt, was offensichtlich im Sinn des Gesetzgebers war, denn die Stärkung des Unternehmenskapitals schien wichtiger zu sein als Ausschüttungen an die Anteilseigner. ${ }^{807}$ Der Gesetzgeber sah sich Anfang des 20.

799 RGBl. 1920, S. 393.

800 Ullmann, Hans-Peter: Der deutsche Steuerstaat, S. 63.

801 Vgl. hierzu Kapital 10.1.2.2.1.

802 Begründung zum Entwurf eines Reichseinkommensteuergesetzes, in: Verhandlungen der Verfassungsgebenden deutschen Nationalversammlung, Bd. 340, Anlagen zu den Stenographischen Berichten, Nr. 1924, Berlin 1920, S. 17.

803 Clauß, J.: Die Körperschaftsteuer, in: Steuer und Wirtschaft. Sondernummer (1922), S. 47-92, S. 54.

804 Evers, Robert: Kommentar zum Körperschaftsteuergesetz vom 10. August 1925, 2. Aufl., Berlin 1927.

$805 \$ 11$ KStG 1920; bei Erwerbsgesellschaften, deren Zweck die Erzielung wirtschaftlicher Vorteile für sich oder ihre Mitglieder war, wurde außerdem auf ausgeschüttete Gewinne unter bestimmten Voraussetzungen bis zum Jahr 1922 eine gestaffelte progressive Zuschlagsteuer erhoben ( $\$ \$ 12$ und $13 \mathrm{KStG} 1920$ ).

806 KStG vom 29. März 1920.

807 Brinkmann, Thomas: Die Körperschaftsteuer, S. 54. 
Jahrhunderts auch in der Pflicht, eine Mehrfachbelastung mit Körperschaftsteuer bei der Verflechtung von Kapitalgesellschaften zu verhindern. In das erste deutsche Körperschaftsteuergesetz wurde als zentrales Rechtsinstitut des Konzernrechts die Privilegierung von Schachteldividenden („Schachtelprivileg“) aufgenommen. ${ }^{808}$

Mit der Einführung des Körperschaftsteuergesetzes 1920 war allein die zivilrechtliche Form für die Besteuerung maßgeblich. Seitdem sind Gesellschaften (juristische bzw. nichtphysische Personen) und Anteilseigner (natürliche bzw. physische Personen) zwei voneinander selbstständige Rechtssubjekte mit der Konsequenz einer strikten Trennung zwischen der Ebene der Gesellschaft und der des Anteilseigners. Dieses Nebeneinander von natürlichen und juristischen Personen als Steuersubjekte wird als duales System bezeichnet und ist eines der prägenden Merkmale unseres heutigen Steuersystems. ${ }^{809}$

1922 wurde eine progressiv abgestufte Vermögensteuer ( $\$ \$ 18-20$ VStG 1922) eingeführt, die als Ergänzungsteuer zur Reichseinkommensteuer angesehen wurde. Auch in diesem Gesetz waren bestimmte subjektive Leistungsfähigkeitsmerkmale berücksichtigungsfähig ( $\$ \$ 21 \mathrm{f}$. VStG 1922).

Der extrem hohe Finanzbedarf des Deutschen Reiches konnte seinerzeit nur mit der zusätzlichen Einführung einer außerordentlichen Kriegsabgabe, einer Vermögenszuwachssteuer und einem „Reichsnotopfer“ beglichen werden. ${ }^{810}$ Die mit dem Reichsvermögensteuergesetz zusammen erlassene Kriegsabgabe für Vermögenszuwächse ${ }^{811}$ sah einen progressiven Steuertarif von 1 bis 10 Prozent des Zuwachses vor. Dabei wurde der zum 30. Juni 1919 erzielte Vermögenszuwachs dem vom 31. Dezember 1913 vorhandenen Vermögen gegenübergestellt. Das Gesetz wurde heftig kritisiert, da es sich mit dem Leistungsfähigkeitsprinzip nicht rechtfertigen lasse. Entscheidend war jedoch, dass diese Steuer mit der Inflation an Bedeutung verlor und sie technisch ad absurdum führte. ${ }^{812}$ Sowohl das Vermögenszuwachssteuergesetz als auch die Wertzuwachssteuer, die Gewinne aus der Veräußerung

808 Ein „Schachtelprivileg“ ist erstmals in $\$ 18$ des Kriegssteuergesetzes vom 21.06.1916 (RGBl. 1916, S. 561 ff.) im deutschen Steuerrecht zu finden; vgl. auch Potthast, Thilo: Die Entwicklung der Körperschaftsteuer, S. 49-53.

809 Jacobs, Otto H./Scheffler, Wolfram/Spengel, Christoph: Unternehmensbesteuerung und Rechtsform. Handbuch der Besteuerung deutscher Unternehmen, 5. Aufl., München 2015, S. 102.

810 Davidsohn, Lars: Verfassungsrechtliche Würdigung, S. 168.

811 Vermögenszuwachssteuergesetz vom 08.04.1922, RGBl. 1922 I, S. 346.

812 Leisner, Walter: Wertzuwachsbesteuerung, S. $50 \mathrm{f}$. 
von solchen Grundstücken, die in der Zeit von 1919 bis 1924 erworben wurden, erfasste, ${ }^{813}$ liefen ohne Verlängerung aus. ${ }^{814}$

Das angesichts „der äußeren Not des Reiches“ im Jahr 1919 eingeführte sogenannte Reichsnotopfer war eine Vermögensabgabe mit einem progressiven Steuerverlauf. Dieser begann mit 10 Prozent für Vermögen bis 50.000 Mark und wies einen Spitzensteuersatz von 65 Prozent bei Beträgen von über 7.000.000 Mark auf. ${ }^{815}$

Neben der Einkommensteuer belasteten direkte Ergänzungsteuern die sogenannten fundierten Einkünfte vorab: die Kapitalertragsteuer, die Grunderwerbsteuer und die Erbschaftsteuer. ${ }^{816}$ Die von Reichsfinanzminister Erzberger als gerecht empfundenen direkten Steuern sollten auf 60 Prozent, dann auf 75 Prozent angehoben werden und die als ungerecht empfundenen indirekten Steuern auf 40 Prozent, dann auf 25 Prozent absinken, jeweils bezogen auf das Gesamtsteueraufkommen. ${ }^{817}$

$\mathrm{Zu}$ der sozialpolitisch orientierten Besteuerungspolitik gehörte auch das im Jahr 1919 eingeführte Erbschaftsteuergeset $z^{818}$ das einen erkennbar umverteilenden Charakter hatte. Der Nachlass des Verstorbenen wurde zunächst mit einem vom Wert des Nachlasses abhängigen Steuersatz in Höhe von 1 bis 5 Prozent vorweg besteuert. Ergänzt wurde diese Nachlasssteuer von einer Erbanfallsteuer, bei der der jeweilige Erbe in Abhängigkeit vom Verwandtschaftsgrad und von der Höhe des Erwerbs mit einem Steuersatz von 4 bis 70 Prozent belastet wurde. Zusätzlich war ein entsprechender Zuschlag zu erheben, der sich nach der Höhe des beim Erben vorhandenen Vermögens bemaß. ${ }^{819} \mathrm{Im}$ Jahr 1925 wurden durch die Neufassung des Erbschaftsteuergesetzes ${ }^{820}$ die Belastungen gesenkt, sodass durch dieses Gesetz keine wesentlichen Umverteilungswirkungen mehr erzielt werden konnten. ${ }^{821}$

813 Gesetz über Änderungen des Finanzausgleichs zwischen Reich, Ländern und Gemeinden vom 10.08.1925, RGBl. I, 1925, S. 254.

814 Leisner, Walter: Wertzuwachsbesteuerung, S. 52.

815 Gesetz über das Reichsnotopfer vom 31.12.1919, RGBl. 1919, \$24, S. 2189 ff.

816 Schremmer, Eckart: Über „gerechte Steuern“, S. 24.

817 Mann, Fritz Karl: Steuerpolitische Ideale, S. 312 f.; Schremmer Eckart: Über „gerechte“ Steuern, S. 24.

818 Erbschaftsteuergesetz vom 10.09.1919, RGBl. 1919, S. 1543 ff, insbesondere hier $₫ 15$ und $₫ 28$.

819 Davidsohn, Lars: Verfassungsrechtliche Würdigung, S. $291 \mathrm{f}$.

820 Erbschaftsteuergesetz vom 22.08.1925, RGBl. I 1925, S. $320 \mathrm{ff}$.

821 Davidsohn, Lars: Verfassungsrechtliche Würdigung, S. 292. 


\subsubsection{Die Korrekturen an der Erzberger-Reform und das Scheitern der Hilferding-Finanzreform}

Die Stabilisierung der Währung, die Klarheit über die Reparationsverpflichtungen und der internationale Kredit trugen dazu bei, dass sich das Wirtschaftsleben in Deutschland wieder normalisierte. ${ }^{822}$

Der 1924 einsetzende Wirtschaftsaufschwung und die verhältnismäßig günstige Finanzsituation der Republik ließen in Wirtschaftskreisen den Wunsch wachsen, dass die Belastung durch Steuern und Abgaben verringert würde. Das veranlasste den Reichskanzler Hans Luther, zunächst dafür zu sorgen, dass die Unternehmen und Besitzenden entlastet wurden. Nachdem er am 15. Januar 1925 Reichskanzler geworden war, nahm er eine Steuerreform in Angriff, wozu Johannes Popitz, der Staatssekretär im Finanzministerium, die entsprechenden Vorarbeiten leistete. Dabei ging es ihm einmal darum, das von Matthias Erzberger geschaffene Steuersystem sowohl den veränderten Gegebenheiten anzupassen, als auch zu konsolidieren, und zum anderen um eine Entlastung der Wirtschaft, damit Kapitalbildung und Investitionen erfolgen konnten. ${ }^{823}$

Die Finanzreform im Jahr 1925 war schon deshalb notwendig, weil die sozialen Folgen des Inflationsprozesses einem Großteil der Bevölkerung erhebliche finanzielle Nachteile beschert hatten und die Steuersätze nicht an den Verlauf der galoppierenden Geldentwertung angeglichen werden konnten. Die Hyperinflation führte dazu, dass das Vertrauen der Bürger in den Staat nachhaltig erschüttert war. Das erklärte Ziel der regierenden Parteien der rechten Mitte war es daher, jeden Einzelnen spürbar zu entlasten und die Umverteilung von Einkommen und Vermögen zu beenden. Insbesondere die Wirtschaft und der Mittelstand sollten geschont werden. Das Ideal der Gerechtigkeit trat damit in den Hintergrund, wurde bei Weitem nicht mehr so stark betont wie noch fünf Jahre zuvor und mit anderen Akzenten versehen. ${ }^{824}$

Vielfach war gegenüber dem EStG 1920 der Vorwurf der Ungerechtigkeit und Ungleichbehandlung erhoben worden. Die unterschiedliche Behandlung der verschiedenen Einkunftsarten war ebenso Ziel der Kritik wie die Überspannung der Steuertarife, die im Zusammenhang mit der Belastung durch andere Steuergesetze beachtet werden mussten. Folglich sollten die Einkunftsarten gleichbehandelt und eine Verminderung der Progression bei der Neugestaltung beachtet werden. ${ }^{825}$

822 Sahm, Reiner: 5000 Jahre Steuern, S. 280.

823 Ebd., S. 281.

824 Hacker, Mark: Gibt es „Gerechtigkeit“ in der Steuerpolitik?, S. 261.

825 Ebd., S. 205. 
Die Steuerbelastung durch die Finanzreform 1919/20 war noch aus einem weiteren Sachverhalt ungleichmäßig und entsprechend ungerecht: Während bei den Lohnsteuerpflichtigen die Lohnsteuer im Quellenabzugsverfahren erhoben wurde und diese damit die neuen Steuern von Anfang an voll tragen mussten, konnten Personen mit hohem Einkommen und großem Vermögen sowie die Unternehmen dem Druck der Steuer ausweichen. Kapitalflucht, Steuerhinterziehung und nicht zuletzt die große Inflation von 1923 eröffneten viele Ausweichmöglichkeiten. Da die Steuern dieser natürlichen und juristischen Personen nicht so zügig erhoben wurden und insbesondere in der Inflationszeit das Einkommen des Vorjahres, dessen Wert die Inflation bereits ausgehöhlt hatte, zugrunde gelegt wurde, entstanden erhebliche Ungleichbelastungen. Wie ungleich der Staat auf den Lohn bzw. das Einkommen oder den Gewinn zugriff, ließ sich daran ablesen, dass im Jahr 192095 Prozent des Aufkommens der Einkommen- und Körperschaftsteuer aus der Lohnsteuer stammten. Im Jahr 1921 waren es rund 60 Prozent, in den Jahren 1922 ca. 75 Prozent und 1923 über 90 Prozent. $^{826}$

Im Einkommensteuergesetz vom 10. August 1925 (EStG 1925) war der Tarifverlauf wie im EStG 1920 progressiv gestaltet, doch waren die Stufen breiter aufgestellt und der Kurvenverlauf deutlich abgeschwächt. Der Höchststeuersatz war mit 40 Prozent um ein Drittel niedriger als im EStG 1920 und galt ab einem zu versteuernden Einkommen von 80.000 Reichsmark ( $\$ 55$ EStG 1925). Um die unteren Einkommen zu entlasten, war ab einem zu versteuernden Einkommen von 12.000 Reichsmark eine weitere Tarifstufe in Höhe von 12,5 Prozent eingefügt worden. ${ }^{827}$

Grundlegend neu war die Einführung einer Besteuerung nach dem Verbrauch gemäß $\$ 49$ EStG 1925. Diese Vorschrift trat ergänzend neben die Besteuerung des Einkommens, wenn das festgestellte Einkommen eines Steuerpflichtigen und sein Verbrauch in einem offenbaren Missverhältnis standen. Dies wurde dann angenommen, wenn der Verbrauch mindestens um die Hälfte höher war als das Einkommen ( $\$ 49$ Abs. 5 EStG 1925). Die Vorschrift fand keine Anwendung, wenn der Verbrauch jährlich weniger als 15.000 Reichsmark betrug oder wenn der Steuerpflichtige nachwies, dass er den Verbrauch aus Vermögen bestritten hatte, das in den letzten drei Jahren der Besteuerung nach dem Einkommensteuergesetz unterlegen hatte ( $\$ 49$ Abs. 6 und Abs. 5 EStG 1925). Dies traf ebenfalls zu, wenn der Verbrauch durch nicht der Einkommensteuer unterliegende Einkünfte bestritten wurde ( $\$ 49$ Abs. 1, 2. Halbsatz EStG 1925). ${ }^{828}$

826 Ullmann, Hans-Peter: Der deutsche Steuerstaat, S. 124

827 Hacker, Mark: Gibt es „Gerechtigkeit“ in der Steuerpolitik?, S. 268.

828 Ebd., S. 245 f. 
Dieser Paragraf stellte im EStG 1925 einen Fremdkörper dar. Ziel dieser Vorschrift war es, Steuersünder zu einer Besteuerung nach dem Einkommensteuergesetz heranzuziehen, auch wenn die Aufnahme dieser Vorschrift unter dem Gesichtspunkt einer modernen Einkommensteuer mit den Grundsätzen einer gerechten Einkommensbesteuerung unvereinbar blieb. ${ }^{829}$

Neben der Einführung einer Besteuerung nach dem Verbrauch hatte das EStG 1925 auch eine erhebliche Zunahme an Paragrafen zur Folge: Während das EStG 1920 noch mit 60 Paragrafen auskam, hatte das neue EStG 1925 mit 117 Vorschriften beinahe doppelt so viele. ${ }^{830}$

Unabhängig vom EStG 1925 wurden auch die Sätze der Vermögen-, Erbschaftund Umsatzsteuern niedriger festgesetzt. Dagegen erhöhten sich - wie es im Dawes-Plan ${ }^{831}$ verlangt wurde - die Verbrauchsteuern für Tabak und Bier. ${ }^{832}$

Am 28. Juni 1928 kam die Regierung der "Großen Koalition“" zustande, in der Rudolf Hilferding das Reichsfinanzministerium übernahm. Das geschah in einer Zeit, in der sich der bisherige Wirtschaftsaufschwung nicht mehr fortsetzte und der Reichsetat im Dezember 1929 ein Defizit von 1,7 Milliarden Mark aufwies. ${ }^{833}$

Da die im Dawes-Plan festgelegten Verpflichtungen sich als eine für das Deutsche Reich nicht tragbare Belastung erwiesen, legte ein Sachverständigenausschuss unter Leitung von Owen Young einen neuen Zahlungsplan für die Reparationen vor, der dem deutschen Wunsch nach Senkung der Schuldenlast entgegenkam. Die Reduzierung der Reparationsraten, die der Young-Plan erlaubte, bot einen Spielraum, um Fehlbeträge zu konsolidieren und die Steuerreform zu finanzieren. ${ }^{834}$

Finanzminister Hilferding und sein Staatssekretär Johannes Popitz unternahmen nun den erneuten Versuch, mithilfe einer Finanzreform zunächst den Haushalt 1929/1930 auszugleichen. Um das zu erreichen, wollten sie folgende Maßnahmen treffen: Ausgabenkürzungen, Steuererhöhungen für Bier und Branntwein, Anhebung der Erbschaft- und Vermögensteuern, Begrenzung des Darlehns für die Arbeitslosenversicherung und Reduzierung der Überweisungen an die Länder.

829 Ebd., S. 247.

830 Strutz, Georg: Das neue Einkommensteuergesetz, in: Steuer und Wirtschaft 4 (1925), S. 1395-1424, S. 1753-1758 und S. 1913-1964, S. S. 1753-1758.

8311924 wurde von der Reparationskommission eine Arbeitsgruppe unter Vorsitz von Charles Dawes einberufen, die auf der Londoner Konferenz vom 16. August 1924 den Dawes-Plan verabschiedete, der die Reparationszahlungen Deutschlands von der deutschen Wirtschaftskraft abhängig machen sollte.

832 Ullmann, Hans-Peter: Der deutsche Steuerstaat, S. 106-109.

833 Sahm, Reiner: 5000 Jahre Steuern, S. 281.

834 Ebd., S. 281. 
Allerdings war der Reichstag für diese Finanzreform nicht zu gewinnen; die Abgeordneten entschieden sich für einen defizitären Haushalt. Außerdem war man sich nicht einig, ob die Arbeitslosenversicherung durch höhere Beiträge oder durch Leistungsreduzierung zu sanieren sei; dazu kam, dass die Länder gegen eine Neuregelung des Finanzausgleiches Widerstand leisteten. ${ }^{835}$

Die Initiatoren dieser Finanzreform sahen keine politische Möglichkeit, durch Notverordnungen ihre Vorhaben zu verwirklichen. Ein Überbrückungsversuch mithilfe eines ausländischen Kredits scheiterte am Einspruch des Reichsbankpräsidenten. Diese Fehlschläge veranlassten Rudolf Hilferding und Johannes Popitz zurückzutreten. Mit großer Wahrscheinlichkeit wäre die geplante Finanzreform wegen der nun einsetzenden Wirtschaftskrise ohnehin nicht von Erfolg gekrönt worden. ${ }^{836}$

\subsubsection{Die Versuche der Staatshaushaltssanierung in den Jahren der Weltwirtschaftskrise}

In den Jahren 1928 und 1929 ließen sich in der deutschen Wirtschaft erste Anzeichen einer konjunkturellen Abschwächung erkennen. Sie deuteten sich in der zurückgehenden Gewinnbildung und im Ausbleiben von Neuinvestitionen an. Als sich aus der Bankenkrise in den USA eine Weltwirtschaftskrise entwickelte, wurde 1930 auch die deutsche Wirtschaft erfasst. Aufgrund einer historisch bedingten besonderen Anfälligkeit kam es einerseits zu einem steilen Abfall des Produktionsund Einkommensniveaus und andererseits zu einer steil ansteigenden Arbeitslosigkeit. Dies wird an der Entwicklung des Nettosozialprodukts zu Faktorkosten und laufenden Preisen besonders sichtbar. Danach sank das Nettosozialprodukt, das in den zurückliegenden Jahren immer wieder zugenommen hatte, 1930 gegenüber dem Vorjahr um 9,0 Prozent. In den Jahren 1931 und 1932 wies es, gemessen an den jeweiligen Vorjahren, einen weiteren Rückgang um 20,6 bzw. um 35,2 Prozent auf. Erst 1933 wuchs das Nettosozialprodukt wieder um 22,1 Prozent. In diesem Jahr lag es aber noch immer 42,9 Prozent unter dem Stand von 1929. ${ }^{837}$

835 Ebd., S. 282.

836 Herzfeld, Hans (Hrsg.): Geschichte in Gestalten, S. 197; Ullmann, Hans-Peter: Der deutsche Steuerstaat, S. $132 \mathrm{f}$.

837 Errechnet nach Hoffmann, Walther G.: Das Wachstum der deutschen Wirtschaft seit der Mitte des 19. Jahrhunderts, Berlin/Heidelberg/New York 1965, S. 509; Sahm, Reiner: 5000 Jahre Steuern, S. 283. 
Die Veränderungen im Wirtschaftsleben der frühen Dreißigerjahre beeinflussten das politische Geschehen in Deutschland und wirkten sich innerhalb kurzer Zeit auf den Staatshaushalt aus. Bis zum Frühjahr 1930 wurde das Land von einer Koalitionsregierung geführt, der der Sozialdemokrat Hermann Müller als Reichskanzler vorstand und die nun genötigt war, die ersten Maßnahmen zur Bekämpfung der Wirtschaftskrise zu treffen. Da durch die rasch ansteigende Arbeitslosigkeit die finanziellen Mittel der Reichsanstalt für Arbeitsvermittlung und Arbeitslosenversicherung abnahmen, galt es zu entscheiden, ob die Mittel aufgestockt oder die Leistungen gekürzt werden sollten. Darüber gab es zwischen den Koalitionspartnern heftige Auseinandersetzungen. Finanzminister Hermann Dietrich und sein neuer Staatssekretär Hans Schäffer sahen den Ausweg darin, die Steuern anzuheben und die Ausgaben zu reduzieren. Dem Vorhaben verweigerte sich der Reichstag, sodass das Kabinett Müller aufgab und am 27. März 1930 zurücktrat. ${ }^{838}$

Nach dem Sturz von Hermann Müller wurde der Zentrumspolitiker Heinrich Brüning am 29. März 1930 zum Reichskanzler ernannt. Das von ihm gebildete sogenannte Frontkämpferkabinett war von Anfang an gewillt, möglichst unter Umgehung des Reichstages mit Notstandsverordnungen zu regieren. Brüning hatte dafür die Unterstützung des Reichspräsidenten, der der Regierung nach Artikel 48 der Weimarer Verfassung erlauben konnte, mit diesem - nur für besondere Fälle gedachten Regierungsinstrument - zu agieren. ${ }^{839}$

Heinrich Brüning machte von diesem Instrument während seiner Kanzlerschaft in der Wirtschafts-, Finanz- und Innenpolitik reichlichen Gebrauch. Er löste schon nach kurzer Amtszeit am 18. Juli 1930 den Reichstag auf, weil die Abgeordneten die von ihm am 16. Juli 1930 eingebrachte Finanzreform nicht mittragen mochten. Damit war die Brüning-Regierung ohne parlamentarische Kontrolle. ${ }^{840}$

Heinrich Brüning verfolgte während seiner Kanzlerschaft im Wesentlichen die drei folgenden Ziele: Sanierung des Staatshaushaltes, Beendigung der Reparationsleistungen und das Wiedererstarken Deutschlands.

Es gelang ihm tatsächlich zu erreichen, dass zunächst die Reparationsleistungen für ein Jahr ausgesetzt wurden. Seinem Wirken war es schließlich mit zu danken, dass die Alliierten am 8. Juli 1932 - bis auf eine Teilzahlung, die ebenfalls nicht mehr aufgebracht wurde - auf weitere Reparationsleistungen verzichteten. Das vergrößerte den finanziellen Spielraum für die Sanierung des Staatshaushaltes. ${ }^{841}$

838 Sahm, Reiner: 5000 Jahre Steuern, S. 284.

839 Ebd., S. 284.

840 Herzfeld, Hans (Hrsg.): Geschichte in Gestalten, S. 197; Ullmann, Hans-Peter: Der deutsche Steuerstaat, S. $132 \mathrm{f}$.

841 Sahm, Reiner: 5000 Jahre Steuern, S. 285. 
Um der während des Krisenverlaufs permanenten Gefahr eines Staatsbankrotts zu begegnen, versuchte es Heinrich Brüning mit unterschiedlichen Maßnahmen auf steuerlichem und finanzpolitischem Gebiet. ${ }^{842}$ Ein ganzes Bündel weiterer Maßnahmen sollte die Staatssanierung unterstützen. Es folgten zahlreiche Anordnungen zur Verringerung der Arbeitseinkünfte: Löhne, Gehälter, Pensionen und soziale Leistungen wurden aufgrund mehrerer Notverordnungen gekürzt.

Durch die vom Kabinett Brüning verfolgte Steuerpolitik erhöhte sich die Steuerlastquote, die das Verhältnis der Einnahmen aus Steuern und Zöllen zum Volkseinkommen ausdrückt. Sie lag 1925 bei 17,6 Prozent, erhöhte sich bis 1928 leicht auf 18,2 Prozent und erreichte 1932 schließlich einen Wert von 24,0 Prozent. ${ }^{843}$

Obgleich die Regierung Brüning in mehreren Notverordnungen Steuererhöhungen verfügt hatte, trat der gewünschte Effekt nur vorübergehend ein. Der Krisenverlauf schmälerte die Fähigkeit der Steuerpflichtigen, die erwarteten Steuersummen überhaupt oder zum nötigen Zeitpunkt aufzubringen, immer weiter. ${ }^{844}$

Dies betraf die Großunternehmen, deren Absatz zurückging, die mittelständischen Firmen, die wegen Auftragsmangels ihre Tätigkeit reduzieren oder teilweise sogar einstellen mussten, oder Kaufleute, deren Kundschaft nicht mehr imstande war, Waren im bisherigen Umfang abzunehmen. Während die Beamten, die Angestellten und die Arbeiterschaft mit einer Arbeitsstelle Steuern entrichten konnten, entfiel der große und ständig wachsende Kreis der Unselbstständigen, die arbeitslos geworden waren, für lange Zeit als Steuerzahler. ${ }^{845}$

Statt das Wirtschaftswachstum und die Schaffung neuer Arbeitsplätze zu fördern, schlug Brüning vor, nicht mehr sanierungsfähige Agrargüter zwangsweise an Kleinbauern aufzuteilen. ${ }^{846}$ Der sich daraus ergebende Konflikt mit den landwirtschaftlichen Großgrundbesitzern führte zum Rücktritt von Brüning am 30. Mai 1932. Dieser Konflikt ging unter dem Begriff „Osthilfeskandal“ in die Geschichtsschreibung ein.

Trotz des großen Bündels an eingeleiteten Maßnahmen - die auch in einer rigorosen Kürzung der staatlichen Ausgaben bestand - erreichten Reichskanzler

842 Vgl. hierzu im Einzelnen: Müller-Mertens, Eckhard u. a. (Hrsg.): Kleine Enzyklopädie Deutsche Geschichte, Leipzig 1965, S. 503-505; Ullmann, Hans-Peter: Der deutsche Steuerstaat, S. 134-136.

843 Petzina, Dietmar: Die deutsche Wirtschaft in der Zwischenkriegszeit, S. 186.

844 Sahm, Reiner: 5000 Jahre Steuern, S. 286.

845 Ebd., S. 286.

846 Köhler, Henning: Arbeitsbeschaffung, Siedlung und Reparationen in der Schlußphase der Regierung Brüning, in: Vierteljahreshefte für Zeitgeschichte 17 (1969), S.27-307, S. $289 \mathrm{f}$. 
Brüning und sein Kabinett das Ziel, den Staatshaushalt zu sanieren, nicht. Es war ihm und seinem Kabinett nicht gelungen, mit den wirtschafts-, finanz- und sozialpolitischen Maßnahmen dem raschen Krisenverlauf zu folgen und damit gleichzeitig die sich zuspitzende politische Situation im Lande zu beherrschen ${ }^{847}$

Mit seiner kontraproduktiven Spar- und Deflationspolitik verstärkte Brüning die Weltwirtschaftskrise und höhlte mit seinen Notverordnungen die Demokratie aus. Andererseits war es ihm zu verdanken, dass die Reparationsverpflichtungen faktisch gestrichen wurden. ${ }^{848}$

\subsection{Die ungelösten Probleme der Finanzpolitik und ihre Auswirkungen auf die finanzwissenschaftliche Forschung}

\subsubsection{Die Entwicklung der zeitgenössischen deutschen Finanzwirtschaftslehre}

Der Erste Weltkrieg, die galoppierende Inflation und die Weltwirtschaftskrise hatten die Finanzpolitik vor völlig neue, teilweise unlösbare Aufgaben gestellt und Wandlungen in einem Ausmaß und einer Plötzlichkeit gebracht wie kaum je zuvor. ${ }^{849}$ Dazu kamen Fragen der Bewältigung der riesigen Verschuldung und der Reparationslasten, nach der Wirkung zu hoher Steuerlasten auf Konjunktur und internationale Wettbewerbsfähigkeit, eine intensive Auseinandersetzung um die unternehmerische Betätigung der öffentlichen Hand und die sich ergebenden Sozialisierungsdiskussionen sowie schließlich das immer deutlicher werdende Beschäftigungsproblem gegen Ende der Zwanziger- und zu Beginn der Dreißigerjahre. ${ }^{850}$

847 Sahm, Reiner: 5000 Jahre Steuern, S. $287 \mathrm{f}$.

848 Hagen Schulze hat die Steuerpolitik der Weimarer Republik kurz zusammengefasst: „Steuerpolitik als Sozialpolitik, als hastiger Notbehelf zur Füllung von Haushaltslöchern, als außenpolitisches Instrument, als Instrument der Domestizierung von Ländern und Gemeinden: alles das gehört zur Geschichte der Steuern in der Weimarer Republik.“ Schulze, Hagen: Die keineswegs Goldenen Zwanziger Jahre, in: Schultz, Uwe (Hrsg.): Mit dem Zehnten fing es an. Eine Kulturgeschichte der Steuer, München 1986, S. 209-218, S. 218 .

849 Scheer, Christian: Die deutsche Finanzwissenschaft, S. 12.

850 Ebd., S. 12. 
Mit den gewaltigen Umwälzungen in Gesellschaft, Staat und Wirtschaft „haben sich auch die Aufgaben der finanzwissenschaftlichen Forschungen geändert, ${ }^{\text {“851 }}$ denn in der Finanzwissenschaft ist ,in der Regel nicht die Theorie den Tatsachen vorangegangen, sondern die Tatsachen haben der Theorie die Aufgaben gestellt und die Wege gewiesen." ${ }^{\text {"852 }}$ Die Vorstellungen über Art und Umfang dieser Veränderungen gingen allerdings weit auseinander. ${ }^{853}$

Nach der sogenannten Edinburgher Regel ${ }^{854}$ war die Finanzpolitik in der ersten Hälfte des 19. Jahrhunderts noch von der „Leave-them-as-you-find-them-rule of taxation" geprägt. Von Teilen der Nationalökonomie ${ }^{855}$ wurde jedoch der Grundsatz, dass die Besteuerung verteilungspolitisch neutral sein müsse, nicht anerkannt. Vor allem in der zweiten Hälfte des 19. Jahrhunderts war der Glaube an das Kräftespiel, der dem klassischen System zugrunde lag, längst erschüttert. Auch aufgrund der auftretenden sozialen Spannungen wurde durch Adolph Wagner der Besteuerung neben der rein finanziellen eine sozialpolitische Aufgabe zugeschrieben.

In den Zwanzigerjahren des 20. Jahrhunderts hatte der Einfluss des Ersten Weltkrieges und der damit verbundenen sozialen Umwälzungen prägenden Einfluss auf das Gesamtbild der Finanzwissenschaft gehabt. Rudolf Goldscheid brachte es auf den Punkt:

Man ist außerstande, exakt Finanzwissenschaften zu betreiben, wenn man nicht beachtet, daß das Gros aller Finanzprinzipien und Finanzpraktiken das Produkt kriegerischer Ereignisse, der Vorbereitung auf Kriege und ihrer Nachwirkung ist. ${ }^{856}$

Das war nicht Neues, bereits 1793 hatte Karl Heinrich von Lang festgestellt: „Es ist mithin ein einleuchtender Satz [...], daß jede Veränderung in dem System der Auflagen oder dem Steuerwesen jederzeit in einer vorausgegangenen Veränderung des Kriegswesens ganz sicher zu suchen ist. ${ }^{\text {(857 }}$

851 Jecht, Horst: Die Entwicklung der Problemstellung in der neueren deutschen Finanztheorie, in: Finanzarchiv 2 (1932), S. 185-240, S. 187.

852 Gerloff, Wilhelm: Die öffentliche Finanzwirtschaft, Vorwort zur Zweiten Auflage.

853 Scheer, Christian: Die deutsche Finanzwissenschaft, S. 13.

854 Mann, Fritz Karl: Steuerpolitische Ideale, S. 246f.; Schremmer, Eckart: Über „gerechte Steuern", S. 15.

855 Vgl. Meyer, Robert: Die Prinzipien der gerechten Besteuerung, S. 410 f.

856 Goldscheid, Rudolf: Staat, öffentlicher Haushalt und Gesellschaft. Wesen und Aufgabe der Finanzwissenschaft vom Standpunkt der Soziologie, in: Gerloff, Wilhelm/Meisel, Franz (Hrsg.): Handbuch der Finanzwissenschaft, Bd. 1, Tübingen 1926, S. 146-184, S. 148 .

857 Lang, Karl Heinrich von: Historische Entwicklung, S. 4. 
Erstaunlich ist, dass in der Nachkriegszeit eine ungewöhnlich große Zahl an zusammenfassenden Darstellungen und auch das von Wilhelm Gerloff und Franz Meisel herausgegebene dreibändige „Handbuch der Finanzwissenschaft“ $(1926-1929)^{858}$ herausgekommen sind, eine ganze Reihe dieser Werke jedoch nur "Grundrisse“ darstellten, und es sich bei den größeren Lehrbüchern mehr oder weniger um aktualisierte Neuauflagen der vor dem Ersten Weltkrieg erschienenen Werke handelte. ${ }^{859}$ Fernerhin erschien eine Vielzahl von Arbeiten, die über eine finanzwissenschaftliche "Standortbestimmung" nicht hinausgingen.

Meisel beklagte, dass die Finanzwissenschaft „die Führung und Kritik der Gesetzgebung aufgegeben ${ }^{\text {“860 }}$ hätte, sie besitze „keinen Zusammenhang mit der Praxis und keinen Einfluß auf die Praxis, weder auf die ministerielle noch die parlamentarische, weder auf die bürokratische noch die der Selbstverwaltung und der öffentlichen Meinung . ${ }^{861}$ In der Politik wurden diese Versäumnisse ebenso gesehen: „Wenn uns nur [...] die neuere Finanzwissenschaft und die Rechtswissenschaft [...] ein besseres Rüstzeug, ja nur Handwerkszeug zur Lösung der steuertechnischen Aufgaben zur Verfügung gestellt hätten." ${ }^{\text {"62 }}$

Erst gegen Ende der Zwanzigerjahre registrierten zeitgenösische Beobachter den Ansatz eines Neubeginns, Zeichen eines neu erwarteten Interesses an der Finanztheorie. ${ }^{863}$

\subsubsection{Die Besteuerung als Instrument der Wirtschaftspolitik: die „Ökonomisierung" der Steuerlehre}

Gegen Ende der Zwanzigerjahre schienen diejenigen an Zahl und Gewicht zu gewinnen, für die eine Finanzpolitik ihren berechtigten Platz neben einer „reinen“ Theorie hatte. Zwar stünde es der Wissenschaft nicht zu, Urteile über die

858 Gerloff, Wilhelm/Meisel, Franz (Hrsg.): Handbuch der Finanzwissenschaft, 3 Bde., Tübingen 1926-1929).

859 Scheer, Christian: Die deutsche Finanzwissenschaft, S. 16-20.

860 Meisel, Franz: Wo steht die deutsche Finanzwissenschaft?, in: Zeitschrift für die gesamte Staatswissenschaft 74 (1919), S. 361-408, S. 394.

861 Meisel, Franz: Stand und Wert der deutschen Finanzwissenschaft, in: Schumacher, Hermann/ Spiethoff, Arthur (Hrsg.): Schmollers Jahrbuch für Gesetzgebung, Verwaltung und Volkswirtschaft im Deutschen Reiche, 42. Jg., Heft 3 und 4, Leipzig 1918, S.317-364, S. 364.

862 Popitz, Johannes: Sammelbesprechung finanzwissenschaftlicher Lehrbücher, in: Jahrbücher für Nationalökonomie und Statistik 116 (1921), S. $556 \mathrm{f}$.

863 Scheer, Christian. Die deutsche Finanzwissenschaft, S. 25. 
Finanzpolitik zu fällen, doch sei es zulässig, für gegebene Ziele Instrumente und Wege aufzuzeigen. ${ }^{864}$

Es traten zwei allgemeine Merkmale hervor: ein Aufleben der Steuerüberwälzungstheorie und das nahezu vollständige Zurücktreten der bis zu Beginn des 20. Jahrhunderts bestimmenden Frage der „Steuergerechtigkeit“ hinter allokative Überlegungen. Die Steuerüberwälzungstheorie fand in der wissenschaftlichen Diskussion bald ihr vorübergehendes Ende, u. a. da sie als unlösbar angesehen wurde. ${ }^{865}$ Demgegenüber forderten die Jüngeren unter den deutschen Finanzwissenschaftlern, „von der ethischen zur ökonomischen Fragestellung ${ }^{\text {“866 }}$ zu gelangen, um das „Glatteis der Subjektivitäten ${ }^{\text {“867 }}$ zu verlassen.

Vor allem aber die Probleme der Kriegsfolgelasten und der Wiederaufbau führten zwangsläufig dazu, sich mit der wirtschaftlichen Seite der Besteuerung unter dem Gesichtspunkt ihrer volkswirtschaftlichen Wirkung und Zweckmäßigkeit zu befassen. Im Gegensatz zu den traditionellen Gerechtigkeitsvorstellungen wurden eine Anregung der Kapitalbildung, eine bewusste Entfaltung der „produktiven Kräfte" der Volkswirtschaft und eine Dämpfung des Konsums für erforderlich gehalten. Nicht ob eine Steuer "gerecht" oder "ungerecht" sei, sondern ob diese die Wirtschaft förderte oder schädigte, wurde zur zentralen Frage erklärt. ${ }^{868}$

Aus den Diskussionen traten zwei neue Steuerideale hervor: Das eine Ideal war der Gedanke einer gezielten steuerpolitischen Maßnahme zur volkswirtschaftlichen Kapitalbildung durch einen gezielten Umbau des Steuersystems. Das zweite Ideal war der Gedanke einer steuerlichen Anspornwirkung der (Unternehmens-) Besteuerung. In der Mehrzahl der herangezogenen Fälle konzentrierte man sich auf den Fall der Rohstoff- bzw. Gerätesteuern, um eine echte „Steuereinholung“869 herbeizuführen, was eine Senkung der Kosten bzw. eine Steigerung der Produktivität gegenüber dem Zustand vor Steuern bewirken würde.

864 Scheer, Christian: Die deutsche Finanzwissenschaft, S. 33 mit Bezug auf Jecht, Horst: Die Entwicklung der Problemstellung, S.233.

865 Moll, Bruno: Lehrbuch der Finanzwissenschaft, Berlin 1930, S. 329.

866 Gerloff, Wilhelm: Steuerwirtschaftslehre, S. 450.

867 Mann, Fritz Karl: Zur Frage der steuerlichen Lastenverteilung. Eine Ergänzung und Entgegnung, in: Jahrbücher für Nationalökonomie und Statistik 124 (1926), S.30-40, S. 34 .

868 Scheer, Christian: Die deutsche Finanzwissenschaft, S. 90-93.

869 Der Begriff wurde durch Schäffle geprägt; Schäffle, Albert Eberhard Friedrich: Die Steuern, S. $309 \mathrm{f}$. 
Die Finanzwissenschaftlerin Dora Schmidt ${ }^{870}$ bemängelte an den Vorstellungen einer „Steuereinholung“, dass diese steuerliche Produktivitätstheorie in hohem Maße von subjektiven Werturteilen abhängig sei, zumal diese Art von Steuerpolitik einseitig die materielle Produktion begünstige. Elsa F. Pfau stand einer differenzierten Behandlung von unterschiedlichen Gewerbezweigen ablehnend gegenüber, da es kaum möglich wäre, objektiv auszuwählen, welche Industrien eine steuerliche Bevorzugung verdienten. ${ }^{871}$ Auch Julius Landmann wies darauf hin, dass die steuerliche Begünstigung eines Industriezweiges regelmäßig die Benachteiligung eines anderen Industriezweiges zur Folge hätte und daher produktionsfördernde Maßnahmen sich am wirksamsten durch dasjenige Steuersystem und diejenige Steuertechnik durchsetzen können, die die Kapitalbildung und die Investitionsrichtung des neu gebildeten Kapitals am wenigsten beeinflussen. ${ }^{872}$

Mit Beginn der Dreißigerjahre wurde die Aufmerksamkeit des finanzwissenschaftlichen Schrifttums zunehmend von anderen Problemen in Anspruch genommen. Konjunkturpolitische Aspekte der Depression fanden mehr und mehr Eingang in die deutsche Finanzwissenschaft. ${ }^{873}$

\subsection{Zusammenfassung und Fazit}

\section{Zum Grundsatz der Allgemeinheit der Besteuerung}

Erstmals in der deutschen Geschichte wurde der Gleichheitsgrundsatz, der ein entscheidendes Kennzeichen der Gerechtigkeit ist, in der Weimarer Verfassung vom 11. August 1919 festgeschrieben. Mit diesem ausformulierten rechtspolitischen Gleichheitsgebot wurden nicht nur die letzten absolutistischen Privilegien, sondern auch alle Arten von unbegründeten steuerlichen Befreiungen und Vergünstigungen für unzulässig erklärt und aufgehoben. Da Verletzungen des Allgemeinheitspostulats meist zugleich Verstöße gegen den Gleichmäßigkeitsgrundsatz und den der

870 Schmidt, Dora: Nichtfiskalische Ziele der Besteuerung. Ein Beitrag zur Steuertheorie und Steuerpolitik, Tübingen 1926, S. $81 \mathrm{ff}$.

871 Pfau, Elsa F.: Industriepolitische Gesichtspunkte in der Besteuerung (Finanz- und Volkswirtschaftliche Zeitfragen 71), Stuttgart 1921, S. 28 f.

872 Landmann, Julius: Produktionspolitische Gesichtspunkte der Besteuerung, in: Colm, Gerhard/ Neisser, Hans (Hrsg.): Kapitalbildung und Steuersystem. Verhandlungen und Gutachten der Konferenz von Eilsen, 1. Teil (Veröffentlichungen der Friedrich List-Gesellschaft e. V. 3), Berlin 1930, S. 171-187, S. 182 und S. 187.

873 Scheer, Christian: Die deutsche Finanzwissenschaft, S. 109. 
Besteuerung nach der Leistungsfähigkeit sind, ${ }^{874}$ war mit der verfassungsrechtlichen Fixierung des Gleichheitspostulats ein Meilenstein gesetzt worden, der einen Jahrhunderte währenden Streit beendet hat.

Als unverzichtbare Grundvoraussetzung einer gerechten Steuerverteilung waren nach dem Grundsatz der Allgemeinheit der Besteuerung alle (natürlichen und juristischen) Personen ohne Rücksicht auf außerökonomische Kriterien zur Steuer heranzuziehen; denn die Befreiung Einzelner von steuerlichen Lasten bedeute in der Regel eine Mehrbelastung anderer. Darüber hinaus enthielt Art. 134 WRV das Gebot, die Steuerlasten auf alle Staatsbürger so zu verteilen, dass diese „im Verhältnis ihrer Mittel" belastet würden. Damit enthielt Art. 134 das verbindliche Gebot, die individuelle Leistungsfähigkeit des Einzelnen angemessen und hinreichend bei der Verteilung der Steuerlasten zu berücksichtigen.

\section{Zum Grundsatz der Gleichmäßigkeit der Besteuerung}

In der Erzberger'schen Finanzreform zeigte sich der Wandel im Staatszweck: Das Steuersystem wurde zu einem sozial- und gesellschaftspolitischen Instrument fortgebildet. Die reichseinheitliche Anwendung des sozialen Prinzips erfasste damit die gesamte deutsche Bevölkerung. Die „Wirkungen der sozialwirtschaftlichen Revolution“, die soziale „Durchblutung“ des Steuerrechts, waren allgemein, ${ }^{875}$ und die grundsätzliche Änderung des Steuer- und Staatszwecks wurde als eine „Anpassung des Steuerrechts an die gesellschaftlich-politischen Vorstellungen " der Zeit gesehen. ${ }^{876}$

Mit dem ersten Reichseinkommensteuergesetz im Jahr 1920 und dessen Weiterentwicklung 1925 beschritt die Politik gänzlich neue Wege, indem erstmals in der deutschen Geschichte eine wirklich moderne und "gerechte" Einkommensteuer vorgelegt wurde. Diese Gesetze berücksichtigten wesentliche Forderungen der Wissenschaft nach einem einheitlichen Aufbau, einer logischen Definition des Einkommens und einer "gerechten“ Besteuerung, die die Grundlage des heutigen Einkommensteuerrechts bilden. ${ }^{877}$

Als mit der konjunkturellen Abschwächung das Beschäftigungsproblem gegen Ende der Zwanziger- und zu Beginn der Dreißigerjahre immer deutlicher wurde und schließlich durch die Banken- und Wirtschaftskrise in den USA auch die deutsche Wirtschaft erfasst wurde, änderten sich auch die Aufgaben der finanzwissenschaftlichen Forschung. Es traten zwei allgemeine Merkmale hervor: ein Aufleben der Steuerüberwälzungstheorie und das nahezu vollständige Zurücktreten der bis dahin

874 Neumark, Fritz: Grundsätze gerechter und ökonomisch rationaler Steuerpolitik, S. 89.

875 Möller, Alex: Reichsfinanzminister Matthias Erzberger, S. 42.

876 Ebd., S. 64.

877 Hacker, Mark: Gibt es „Gerechtigkeit“ in der Steuerpolitik?, S. 397 und S. 275. 
bestimmenden Frage der „Steuergerechtigkeit“ hinter allokative Überlegungen. Im Gegensatz zu den traditionellen Gerechtigkeitsvorstellungen wurden eine bewusste Entfaltung der "produktiven Kräfte" der Volkswirtschaft und eine Dämpfung des Konsums für erforderlich gehalten. Das Scheitern der Selbstheilungskräfte der Wirtschaft hatte nachhaltig den Staatszweck, die Budgetregeln und die Funktion der Steuer verändert. Es wurde nicht mehr gefragt, ob eine Steuer "gerecht" oder „ungerecht" war, sondern ob diese die Wirtschaft förderte oder schädigte.

Damit hatte sich das Jahrhunderte bestehende Steuerideal radikal geändert, was Fritz Karl Mann zu folgender Feststellung veranlasst hatte:

Es gehört zu den erstaunlichsten Tatsachen der modernen Finanzgeschichte, daß das neue steuerpolitische Ideal - trotz seiner Verleugnung von Herkommen und Gewohnheit - im Sturm die Geister erobert hat. Nur der Siegeszug der Universalakzise des 17. Jahrhunderts stellt einen vergleichbaren Vorgang dar. ${ }^{878}$

Der Grundsatz der Gleichmäßigkeit der Besteuerung, nach dem die Steuerpflichtigen nicht nur rechtlich, sondern auch tatsächlich nach ihrer Leistungsfähigkeit belastet werden sollten, wurde mit diesem Wandel „von der ethischen zur ökonomischen Fragestellung" teilweise bewusst und planmäßig außer Kraft gesetzt. ${ }^{879}$ So erschien ein Konflikt zwischen der Sozialpolitik und der produktivistischen Steuerpolitik unvermeidlich ${ }^{880}$ was zu einem Widerstreit zwischen definierter Steuergerechtigkeit gemäß Verfassung und angestrebter Wirtschaftspolitik gemäß dem Willen des Parlaments führen sollte. ${ }^{881}$

\section{Zum Prinzip der Besteuerung nach Leistungsfähigkeit}

Mit der sozialpolitischen Aufgabe hatte die Steuer ihre wirtschafts- und sozialpolitische Bedeutung erhalten. Der konkrete Inhalt der "gerechten“ Steuer hatte sich auch durch die Umdeutung des Steuerverteilungskriteriums „Leistungsfähigkeit“ gewandelt. Diese Wandlung hatte zur Folge, dass nicht mehr die Steuer als solche gerecht sein sollte, sondern das soziale Ziel, das mit der Steuer erreicht werden sollte, wurde als gerecht empfunden.

Dass die Leistungsfähigkeit der wichtigste Indikator der steuerlichen Gerechtigkeit ist, hob Matthias Erzberger in seiner Rede vor der Nationalversammlung am 8. Juli 1919 hervor: „gerechte Steuern erfassen jeden nach seiner Leistungs-

878 Mann, Fritz Karl: Steuerpolitische Ideale, S. 349.

879 Davidsohn, Lars: Verfassungsrechtliche Würdigung, S. 306.

880 Mann, Fritz Karl: Steuerpolitische Ideale, S. 348 f.

881 Schremmer, Eckart: Über „gerechte Steuern“, S. 23. 
fähigkeit“" 882 Diese Feststellung schlug sich insbesondere in den Regelungen des EStG 1920 bezüglich steuerfreier Einkommensbestandteile ( $\$ 12)$, der Einführung einer Haushaltsbesteuerung ( $\$ \$ 16,17)$, der Berücksichtigung eines steuerfreien Existenzminimums ( $\$ 20)$, im Steuertarif $(\$ 21)$ sowie bei der Berücksichtigung verschiedener wirtschaftlicher Verhältnisse, die die Leistungsfähigkeit des Steuerpflichtigen wesentlich beeinträchtigen $(\$ 26)$, nieder.

Die Finanz- und Steuerreform von Erzberger bestand in einer Reihe von neuen und veränderten Steuergesetzen, bei denen eine soziale Komponente zugrunde gelegt wurde. Die Einkommensteuer bildete dabei jedoch den Schwerpunkt innerhalb des Gesamtsteuersystems, da bei dieser in besonderem Maße die steuerliche Leistungsfähigkeit berücksichtigt werden konnte und auch weniger erwünschte Belastungswirkungen anderer Steuerarten weitgehend kompensiert werden konnten. ${ }^{883}$

Matthias Erzberger setzte die sozial- und steuerpolitischen Forderungen der Weimarer Parteien nach 1919 nur teilweise um. Seine Finanzreform erwies sich dennoch formell als Erfolg und „markierte eine Zäsur in der Geschichte der Finanzen“" ${ }^{884}$ Materiell hingegen verfehlte die Reform ihr Ziel. Die hohen Einkommen- und Vermögensteuersätze stießen auf heftigen Widerstand bei den Betroffenen, Kapitalflucht und Steuerhinterziehung nahmen zu. Dazu kam, dass die Steuersätze nicht so flexibel gestaltet waren, dass sie sich der Inflation anzupassen vermochten; „die Inflation galoppierte der Steuerreform samt allen ihren Nachbesserungen davon“ ".885

Das EStG 1925 war gegenüber dem EStG 1920 systematisch und strukturell verbessert worden. Grundlegende Vorschriften wurden nahezu unverändert übernommen, die Regelungsdichte wurde erhöht, die Paragrafenzahl nahezu verdoppelt. Das Gesetz sollte eine umfassende Rechtsgrundlage bieten, um dem Bürger Planungssicherheit für seine wirtschaftlichen Dispositionen zu geben. Die Kernforderungen der modernen Einkommensbesteuerung - Besteuerung des gesamten tatsächlichen Nettoeinkommens unter Berücksichtigung der persönlichen Verhältnisse - wurden erfüllt und waren Vorbild für unser heutiges Einkommensteuerrecht.

\section{Zum Redistributionspostulat oder zum Grundsatz der steuerlichen Umverteilung von Einkommen und Vermögen}

Ein konkreter Tarifverlauf oder eine konkrete Höhe der Steuerbelastung wird durch das Prinzip der Besteuerung nach der Leistungsfähigkeit selbst nicht vorgeschrieben. Das Progressionspostulat ist jedoch grundsätzlich mit dem Leistungsfähig-

882 Erzberger, Matthias: Reden, S. 5.

883 Vgl. hierzu Wagner, Adolph: Finanzwissenschaft 2. Teil, $₫ 155$, S. 370 .

884 Ullmann, Hans-Peter: Der deutsche Steuerstaat, S. 103.

885 Ebd., S. $103 \mathrm{f}$. 
keitsgrundsatz untrennbar verbunden, denn jede konkrete Progressionsgestaltung enthält ein kleineres oder größeres Maß an Willkür, das die jeweils herrschenden ökonomisch-politischen Ideologien und Machtpositionen widerspiegelt. ${ }^{886}$ Es stellt sich daher die Frage, ob im gesamten Bereich der Progressionsskala im Einkommensteuergesetz vom 29. März 1920 eine weitgehend gleichmäßige und verhältnismäßige Steuerlastverteilung gewährleistet war. Aus mathematischer Sicht wurde der Kurvenverlauf kritisiert, da die Leistungsfähigkeit sich nicht in dem Stufengrenztarif niederschlug. Paul Riebesell vertrat den Standpunkt, dass aufgrund der erheblichen Sprünge in dem Stufengrenztarif die „bereits erlassenen Gesetze hinsichtlich der Tarife dringend der Revision" bedürften. ${ }^{887}$

Das EStG 1920 war sehr stark von dem enormen Finanzbedarf des Reiches nach dem Ersten Weltkrieg beeinflusst, was auch seinen Niederschlag in der amtlichen Begründung fand. Allgemeiner Konsens bestand darin, dass Kriegsgewinne abgeschöpft werden sollten - was mit der Einführung einer außerordentlichen Kriegsabgabe, einer Vermögenszuwachssteuer und einem „Reichsnotopfer“ geschah - und dass jeder Steuerpflichtige zur Verminderung der Lasten beitragen musste.

Unabhängig von diesen außerordentlichen Steuern belasteten direkte Ergänzungsteuern die sogenannten fundierten Einkünfte: die Kapitalertragsteuer, die Grunderwerbsteuer und die Erbschaftsteuer. Die Erbschaftsteuer hatte einen deutlich erkennbaren umverteilenden Charakter.

886 Neumark, Fritz: Grundsätze gerechter und ökonomisch rationaler Steuerpolitik, S. 179.

887 Riebesell, Paul: Die neuen Reichssteuertarife vom mathematischen Standpunkt aus. Vortrag, gehalten in der Abteilung Mathematik auf der 86. Versammlung Deutscher Naturforscher und Aerzte in Bad Nauheim, in: Zeitschrift für die gesamte Staatswissenschaft 75 (1921), S. 469-477, S. 469-472 und S. 477. 
Dieses Buch wird unter der Creative Commons Namensnennung 4.0 International Lizenz (http://creativecommons.org/licenses/by/4.0/deed.de) veröffentlicht, welche die Nutzung, Vervielfältigung, Bearbeitung, Verbreitung und Wiedergabe in jeglichem Medium und Format erlaubt, sofern Sie den/die ursprünglichen Autor(en) und die Quelle ordnungsgemäß nennen, einen Link zur Creative Commons Lizenz beifügen und angeben, ob Änderungen vorgenommen wurden.

Die in diesem Buch enthaltenen Bilder und sonstiges Drittmaterial unterliegen ebenfalls der genannten Creative Commons Lizenz, sofern sich aus der Abbildungslegende nichts anderes ergibt. Sofern das betreffende Material nicht unter der genannten Creative Commons Lizenz steht und die betreffende Handlung nicht nach gesetzlichen Vorschriften erlaubt ist, ist für die oben aufgeführten Weiterverwendungen des Materials die Einwilligung des jeweiligen Rechteinhabers einzuholen.

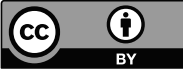

